\title{
How to Integrate Language Concepts to Build a Program: A Literature Review
}

\author{
R.Sangeeta \\ M.Tech CSE, Vishakhapatnam, Andhra Pradesh, India
}

\begin{abstract}
This paper is about how understanding of language concepts helps to integrate and build a program. In this paper we will discuss how small techniques, concepts are put together. We will take examples and show how to build a program.This is to make understand to focus on major language concepts from where programming actually starts. Actually many upcoming programmers struggle to understand this simple concept.
\end{abstract}

Keywords -Integration, C concepts, Program, Building program.

\section{INTRODUCTION}

There is a wide gap between understanding a concept, learning, putting it together.Although many new concepts are emerging focusing on new technologies but are we making much of it. More understandable and practicable. Here i am going to put forward techniques, learning concepts and Integrating.

There is a standard structure for writing a program in particular language. We can relate a day to day activity with a program. For example activity includes some task where we follow its procedure to complete it. In the same way program has to be written by following syntax of that language. Starting with $\mathrm{c}$ language we have many languages features in c language. Starting from declaration, inclusion of header files, structure, pointers and understanding statements to execute program.

Our initial concept is how to declare variables, write simple executable statements to make comfortable to understand the structure.As we progress we focus on loops which helps to perform repetitive operations fast. Next comes operators helps to perform mathematical functions. Array to store set of data which are of same data type. Structures, Pointers are the most important features of c language. Finally if we observe structure which is a collection of heterogeneous data types. So our program or project might start with structure. Slowly we are adding or building basic concepts to perform a big task.

As we progress oops concept has a major impact on programming. It has changed the style of programming. Here we will cover topics related to object oriented programming. I want to put focus on these simple concepts. How it is build and integrated in a program. I want to make students and new upcoming programmers to feel comfortable about programming. If this is made clear it may improve their programming skills, set a stage for them to implement new technologies efficiently.

\section{BASIC LANGUAGE CONCEPTS}

In order to achieve the purpose of research objective how many basic language elements we use to build a program we will consider an example of ' $\mathrm{C}$ ' Program. Variable:

\section{VARIABLE AND ARRAY}

A variable can store only one value of particular data type. If we want to use variable we need to declare it first in the program. Then we need to follow the rules of variable declaration like it cannot be a keyword, first letter should not begin with alphabet etc. [2].

For e.g. int a;

Array: To store a set of values of same data type we can use array.Here we need to understand how to read and write arrays using loops.Its important to remember about dimension or size of array.

For e.g. int a[10] ;

Here a is the name of array, int is the data type which holds 10 integer values starting from 0 to 9 . 
Consider the following simple program to demonstrate how variables are declared, read a value using scanf, printf using printf and use of if else statement.

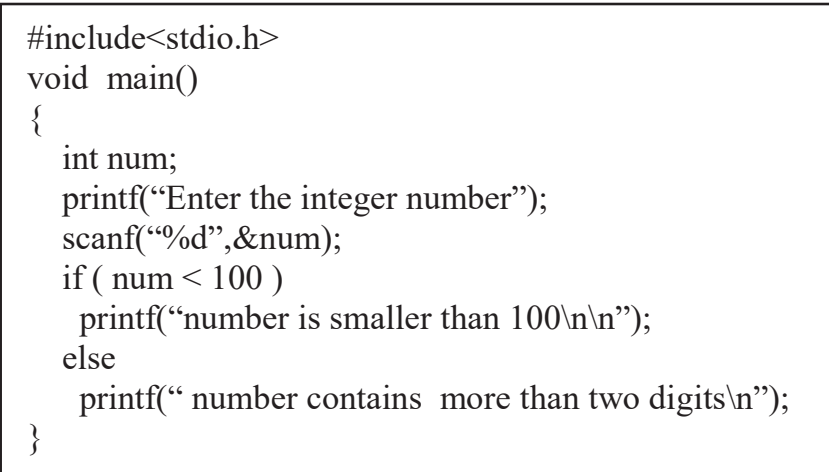

Figure1. Simple C program

\section{STRUCTURE}

If we carefully observe the following example of structure which is studied at the end. It is built with basic language features, the individual datatypes which are declared individually in the beginning are enclosed in the structure as structure members i.e. group of dissimilar data types into one data type i.e. structure. Here char name, int age, int id, togetherely stands for full description of employee.

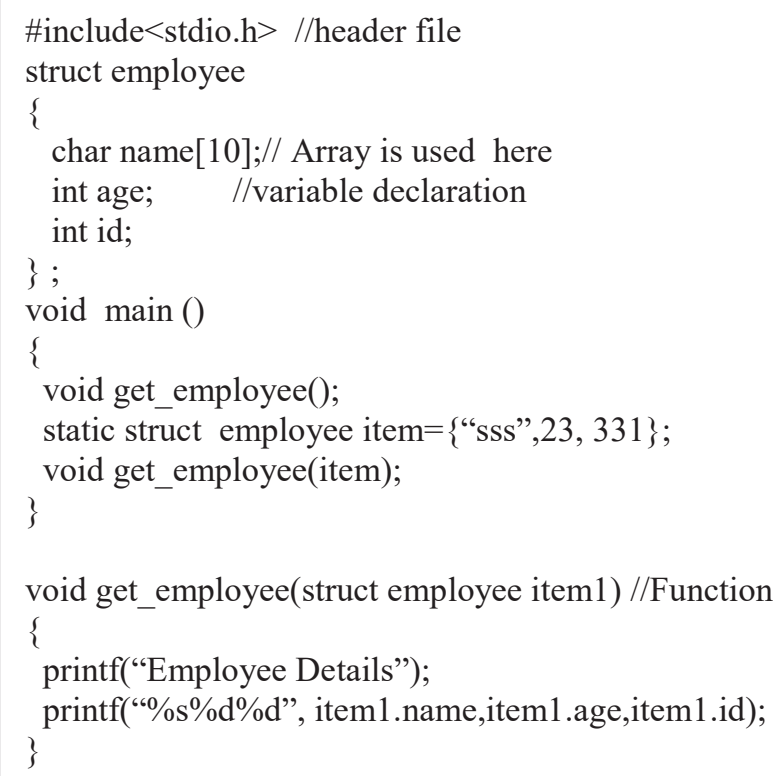

Figure2. Program to illustrate structure

\section{FILE}

Consider another important concept of "C" File .There are built in functions to open, to close, operate or perform operations on files. File concepts are used fopen(), fclose(), putc(), getchar() different functions are called to save the data in the disk. We have also used the concept of function. If we think of working on files its logical .To makes it 
really work we need to understand its functionality and integrate its concept together. So small built in functions are helping to save our files on to disks.

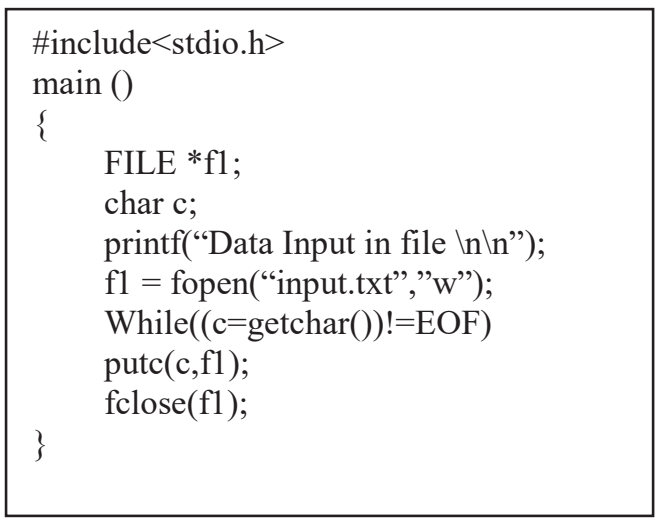

Figure3. Program to illustrate File

\section{OBJECT ORIENTED PROGRAMMING}

This has changed the style of programming where we can program the real world object. Here we have the concept of class where we declare members and member functions. The same concept can be applied to class programs also. There are many examples to understand. Initially we work with only one class but as the complexity of the program to build software increase we need subclass, Abstract class, Interface. There is purpose of each concept if we understand this concepts then learning programming or building programs is much more innovative and interesting.

\section{CONCLUSION}

In this paper i have put forward some available techniques and shown how integration of language concepts helps to build a program. I have widen the concept in much more technical way with examples. I would like to conclude that program is written from basic language features only just we need to think on how to program effectively by integrating all the language features.

\section{REFERENCES}

[1] V. S. Handur, P. D. Kalwad, N. Yaligar, V. G. Garagad and M. K. Pawar, "An activity based learning: C programming," MOOCs, Innovation and Technology in Education (MITE), 2015 IEEE 3rd International Conference on, Amritsar, 2015, pp. 310-314. doi: 10.1109/MITE.2015.7375336

[2] [Stroustrup 86] B. Stroustrup, The C++ Programming Language, Addison-Wesley: Reading, Mass., 1986. Second edition, 1991. 